\title{
Advanced Mechanical Circulatory Support With the HeartMate Left Ventricular Assist Device in the Year 2000
}

\author{
James W. Long, MD \\ Utah Artificial Heart Program, LDS Hospital, Salt Lake City, Utah
}

This paper describes the HeartMate left ventricular assist device (ThermoCardiosystems Inc, Woburn, MA) technology, which has been successfully introduced into the clinical arena with more than 2,400 implants as of the year 2000. The review summarizes the clinical experi- ence, and identifies the benefits and limitations of the current state-of-the-art technology of the leading implantable circulatory support system.

(Ann Thorac Surg 2001;71:S176-82)

(C) 2001 by The Society of Thoracic Surgeons

$\mathrm{T}$ he leading implantable circulatory support technology today is the pulsatile, TCI HeartMate (ThermoCardiosystems Inc, Woburn, MA) left ventricular assist device (LVAD). Thirty-five years after original research began paving the way for this technology and 15 years after it was first used in patients, the HeartMate has been successfully introduced into the clinical arena with more than 2,400 implants as of the year 2000.

The field of advanced circulatory support has matured with the evolution of the HeartMate. Nearly 170 centers worldwide have used this technology since a handful of centers conducted the first clinical trials starting in 1985. No other advanced circulatory support technology has been this well accepted. Advancement of the therapy and science of advanced mechanical circulatory support as well as experience to guide the development of nextgeneration technologies are outcomes of pioneering clinical experience with the HeartMate. Even as nextgeneration heart pumps are being introduced into the clinical arena, the first-generation HeartMate is still making important contributions to the field by being evaluated for long-term therapy in the landmark clinical trial REMATCH in the US.

An overview of the HeartMate LVAD technology and the clinical experience with this device are presented to identify the benefits and limitations of the current stateof-the-art technology.

\section{Material and Methods}

\section{HeartMate Technology}

The HeartMate LVAD is a pulsatile, implantable blood pump made of titanium with a polyurethane diaphragm backed by a pusher plate actuated by either an external

Presented at the Fifth International Conference on Circulatory Support Devices for Severe Cardiac Failure, New York, NY, Sept 15-17, 2000.

Address reprint requests to Dr Long, Utah Artificial Heart Program, LDS Hospital, 324 Tenth Ave, \#160, Salt Lake City, UT 84103; e-mail: jimlong@ihc.com.

pneumatic driver (the IP, Implantable Pneumatic version) or by an internal electric motor (the VE, Vented Electric version) [1, 2].

BLOOD PATH. The blood pump is interposed between the left ventricular (LV) apex and the ascending aorta. The $\mathrm{LV}$ apex is cannulated by removing a circular plug of myocardium and securing a sleeve conduit with sewing ring to the opening and, then, passing the LVAD apical uptake conduit through the sleeve into the LV. Blood enters the pump through a Dacron conduit in which is suspended a $25-\mathrm{mm}$ porcine inflow valve; a similar valve conduit exists on the outflow side. Blood is returned through a $20-\mathrm{mm}$ woven Dacron outflow graft anastamosed to the proximal ascending aorta.

TEXTURED BLOOD CONTACTING SURFACES. The blood pumping surfaces are textured with sintered titanium microspheres on the titanium surfaces and an integral fibrillar texture on the polyurethane diaphragm. This unique, textured surface, the only textured blood pump surface in use clinically, promotes a thin, adherent coagulum that organizes into a biological matrix. This neointimal surface is composed of acellular elements, mostly collagen, and cells derived from circulating progenitor cells differentiating into fibroblasts, myofibroblasts, monocytes, and even endothelial cells. This host-synthesized neointimal lining remains thin, avoiding excessive hyperproliferative deposition or pannus formation. The combination of this bioengineered, hybrid surface, the use of tissue valves, and well-engineered blood flow pathways produces a biocompatable interface for blood. The result is such a low propensity for thrombus formation that only low-level antiplatelet anticoagulation with aspirin is required. The interesting composition of this surface, and its hematologic and immunologic properties have been extensively studied [3-7].

CHARACTERISTICS. The original HeartMate, the pneumatic version (IP), is powered by one of three sources: (1) a 
console-based driver weighing 73 lbs supported on a push cart with wheels; (2) a portable driver (PortPak) weighing $20 \mathrm{lbs}$; or (3) a small hand pump as backup for emergencies. Pulses of air actuate the pusher plate diaphragm within the implanted LVAD, passing through a percutaneous driveline, $1.2 \mathrm{~cm}$ in diameter. The driveline is covered with a textured, polyester velour sheath that accommodates tissue incorporation to create a barrier against ascending infection. The IP blood pump is $11.2 \mathrm{~cm}$ in diameter and $4 \mathrm{~cm}$ thick.

The advanced electric system (VE), brought to clinical use after the IP, is powered by an internal, electric motor that rotates once for each pulse, during which a ramped cam mechanism displaces the pusher plate of the diaphragm. Air displaced by the movement of the diaphragm within a nondisplaceable housing is vented to the atmosphere through a percutaneous vent line. The VE version of the blood pump is $11 \mathrm{~cm}$ in diameter and $4.0 \mathrm{~cm}$ thick, weighs $1190 \mathrm{~g}$, and occupies $460 \mathrm{cc}$. Power is supplied by two external video camera-size batteries (gel cell type lead acetate, $18 \times 9 \times 2.5 \mathrm{~cm}, 650 \mathrm{~g}$ each) coupled to an external controller about the size of two pagers $(8.6 \times 8.0 \times 2.3 \mathrm{~cm}, 260 \mathrm{~g})$. The external controller is connected to the internal pump through a percutaneous lead, textured as with the IP. Two batteries provide power for 4 to 7 hours depending on age of the batteries and pumping conditions. The vent line is incorporated into the percutaneous lead and serves the dual purpose of venting or pneumatic actuation. Because the diaphragm of the VE pump can move independently of the motor drive system, it can be actuated pneumatically as well as electrically. A pneumatic driver or emergency handpump can be connected to the externalized vent line and used as an alternative drive system, providing safety backup in the event of failure of the electrical drive system. Additional components include a bedside power base unit for charging batteries and providing a direct source of electric power, and an auxiliary digital display and control unit for use in the medical environment. A 24-hour emergency battery is available. Ergonomic accessories include harnesses for the externals and a waterresistant covering for the percutaneous lead so that a shower can be taken.

OPERATION. The blood pumping chambers and pathways of both versions of the HeartMate have a maximum effective stroke volume of $83 \mathrm{cc}$. The control system can operate in the fixed-rate mode or an autoregulating mode. In the auto mode, stroke volume is maintained at an effective $78 \mathrm{cc}$ while rate is varied in response to preload.

The VE can pump between 50 and $120 \mathrm{bpm}$, varying pump flow output from 4 to $10 \mathrm{~L} / \mathrm{min}$. In the fixed-rate mode, adjustable between 50 and $120 \mathrm{bpm}$, stroke volume depends on filling. The rate should be manually adjusted so that stroke volumes are 70 to $80 \mathrm{cc}$. Stroke volumes persistently lower than this result in undesirable flow characteristics risking thromboemboli, especially if operated at less than $30-\mathrm{cc}$ stroke volumes. The HeartMate pumps operate independently of native heart function, asynchronous of native heartbeat. An option for synchronous pumping, originally engineered into the pump, has not been needed clinically, although weaning to recovery may be better served by synchronous operation.

One characteristic of operation, especially important while weaning from cardiopulmonary bypass (CPB), is that the VE controller does not adjust to rates below 50 bpm, even when filling volume is low. Under such conditions of low filling volumes, while the pump is still running at its minimum rate, negative suction pressures (about minus $10 \mathrm{~mm} \mathrm{Hg}$ ) can occur within the blood path of the pump, generated as the polyurethane diaphragm, manufactured with inherent tension loading, returns to its state of full relaxation. When negative pressures occur, it is possible to aspirate air through junctions and graft interstices, resulting in air embolization.

AVAILABILITY AND REgUlatory STATUS. The HeartMate IP pneumatic LVAD was the first implantable blood pump in the US to receive Food and Drug Administration approval, granted in 1994 for the application of bridging to transplantation. The electric HeartMate VE, received similar approval in 1998. Further applications for this technology, currently undergoing clinical trial, include an evaluation of the HeartMate VE versus optimum medical therapy as alternatives to transplantation. This randomized, prospective trial, the REMATCH trial (Randomized Evaluation of Mechanical Assistance for the Treatment of Congestive Heart failure), sponsored by the National Institutes of Health, is the first of its kind in the US to evaluate an implantable blood pump for long term therapy [8].

The HeartMate technology is available internationally, in 24 countries, in North America, Europe, the Middle East, South America, and the Asia Pacific area.

\section{Clinical Implementation}

The HeartMate is designed to provide mechanical circulatory support for patients with compromising hemodynamic deterioration. Typically, these patients have low cardiac output, low blood pressure, and elevated cardiac filling pressures of sufficient magnitude to compromise end organ function or activities essential for life.

The patient population in which the HeartMate has been used include transplant candidates who deteriorate before a donor becomes available; patients with threatening, irrecoverable, hemodynamic compromise, chronic or acute, who are expected to be eligible for transplantation when medically stabilized; patients with hemodynamic deterioration, chronic or acute, whose hearts may recover after a extended period of support; and patients who have no hope of recovery or transplantation.

Bridging to transplantation with the HeartMate is proved therapy. Bridging to recovery and "permanent" therapy ("destination therapy" or alternative to transplantation) are evolving indications. Experience with chronic cardiomyopathies, ischemic or idiopathic, exceeds that with acute cardiac dysfunction.

Patients of small size are excluded. The recommended, 
allowable size is body surface area (BSA) $\geq 1.5$, although it has been used in patients with a BSA of $1.3 \mathrm{~m}^{2}$. Although generally used in adults, implantation of the HeartMate in large adolescents can be undertaken [9].

Contraindications other than size are relative, based on likelihood of success. Early intervention, before the onset of multiorgan dysfunction, is the most important factor influencing outcome. Major risk factors include hepatic, renal, or pulmonary failure, active infection or susceptibility, and technical challenges such as right heart dysfunction and redo operations. Screening scales have been established for predicting outcome based on preoperative risk factors [10].

\section{Implantation and Management}

Preoperative planning optimizes the ergonomics of the pump and percutaneous lead placement. Wide-spectrum antimicrobial prophylaxis is used, anticipating short duration exposure.

The HeartMate device is prepared by preclotting the Dacron-valved conduits (inflow and outflow) as well as the Dacron return graft. The pump body is placed in the left upper quadrant, either in a preperitoneal pocket [11] or intraabdominal [12]. Preferences for pump position are guided by considerations about ease of explant and fear of intraabdominal complications such as bowel or organ injury for those using the preperitoneal approach, or concern about preperitoneal pocket infections for those using the intraabdominal approach. An opening across the left anterior diaphragm into the apex of the pericardium is created for the inflow conduit. The outflow conduit and aortic graft are directed just to the right of midline to avoid reentrant problems. The percutaneous lead courses from the left side of the pump body, in curvelinear fashion, to the right upper quadrant exiting just below the right costal margin in the midclavicular line. The external portion of the percutaneous lead is directed towards the right axilla, allowing immobilization against the rigid lower thorax while achieving acceptable ergonomics. Immobilization is vital for tissue incorporation at the exit site, essential to prevent "ascending" infection migrating along the percutaneous leads towards implanted components.

The Dacron return graft is anastomosed to the proximal ascending aorta to the right of its centerpoint. Cardiopulmonary bypass, preferably without cross-clamp to avoid right heart ischemia, is employed while the LV apex is cannulated after a core of myocardium has been removed and a sleeve with a sewing ring has been sutured in place.

Concurrent procedures, as necessary for proper and safe LVAD performance, are performed: removal of LV thrombus, closure of patent foramen ovale (PFO), correction of aortic valve insufficiency or mitral stenosis, and right heart coronary bypass. Other procedures, perhaps useful for long-term management are considered, such as: replacement/obliteration of prosthetic valves with thromboembolic potential, and correction of mitral or tricuspid regurgitation.

Deairing is completed. Right heart function is opti- mized using inotropes, pulmonary vasodilator(s), and correction of arrhythmias and metabolic, acid/base imbalances. Appropriate volume loading is essential, avoiding underfilling the left heart/LVAD (risking suction and air embolization) or distending the right heart.

Weaning from CPB is undertaken, cautiously. Careful monitoring during this process is important; transesophageal echo is essential and left and right atrial pressure monitoring is useful. It has been recommended that cardiopulmonary bypass (CPB) blood be shunted into the left heart during the early phases of weaning to assure adequate loading of the LVAD to deliver good systemic perfusion and pressure, allowing the right heart to be gradually loaded in a controlled manner as this "shunt" into the left heart is decreased [13]. Even with appropriate left heart and LVAD filling, it is desirable to start the VE LVAD pneumatically, using a hand pump or a pneumatic console connected with sterile tubing to the VE vent, to allow low flow rates during the startup.

Infection precautions perioperatively are continued with wide-spectrum antimicrobial use for 48 hours, after which, further antimicrobial therapy is targeted to infection or narrow spectrum prophylaxis until tubes and lines are out. Meticulous sterile technique is obligatory with management of wounds, tubes, and lines. Meticulous percutaneous lead and exit site care is critical. Cleanliness and immobilization must be assured. An elastic, immobilizing binder, wrapped around the torso, to which the percutaneous lead can be secured, seems to work best.

Anticoagulation requirements are generally limited to aspirin with or without Persantine. Coumadin is recommended only for non-LVAD indications.

\section{Results}

\section{Clinical Experience}

Experience with the HeartMate continues to increase annually. In 1999, 440 HeartMate LVADs were implanted, three times more than in 1994. Figure 1 demonstrates the annual growth in the use of HeartMate LVADs.

Although the numbers of HeartMate LVADs implanted continue to increase, only a small proportion of the population that could conceivably benefit from advanced mechanical circulatory support is being served. As long as the major indication for the HeartMate remains bridge to transplantation, experience will be limited to a proportion of the worldwide transplant experience of 3,300 transplants per year. The REMATCH trial is studying appropriate therapy for nontransplantable heart failure patients.

The number of centers worldwide using the HeartMate continues to increase, to a total of 170 centers in the year 2000. This is more than twice the number of centers in 1995, when 80 were involved. The annual growth in number of centers is shown in Figure 1.

As of the year 2000, there are 112 centers in North America, 37 in Europe, 13 in Asia Pacific, 5 in the Middle East, and 3 in South America. Eighty-four percent of the 


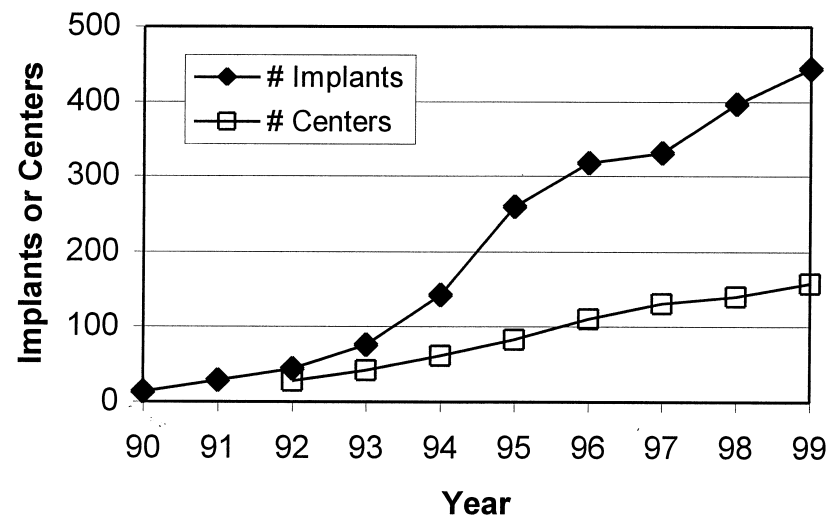

Fig 1. Number of HeartMate implants and number of centers by year.

HeartMate LVADs used clinically have been implanted in the US.

The widespread dissemination of the HeartMate technology into the hands of a large number of advanced heart failure centers around the world parallels growth of the field. It will be challenging to maintain expertise during this early growth phase. Many centers will not implant more than two HeartMate LVADs per year until indications expand and then volumes increase.

\section{Clinical Outcomes}

CLINICAL TRIALS. The clinical trials of the HeartMate devices established their efficacy and safety for bridging to transplantation. The clinical trial of the pneumatic HeartMate IP as a bridge to transplantation was conducted from 1985 to 1993 . It was a controlled trial with 116 LVAD patients and 46 control patients.

Effectiveness measures included survival, hemodynamic performance, and end-organ response. The probability of survival to transplantation was significantly better in LVAD patients (LVAD patients had a $58 \%$ probability of surviving at 11 months after implant, whereas control patients had only a $9 \%$ chance of being alive at only 2 months). LVAD patients also had a greater probability of survival posttransplantation (LVAD patients had a $90 \%$ probability of being alive 1 year after transplantation compared with control patients who only had a $67 \%$ chance of survival). Hemodynamic performance and end-organ recovery were improved by LVAD support.

Safety analysis of all adverse events was conducted. Even with conservative definitions, only $25 \%$ of adverse events were considered device related. The devicerelated rate of adverse events included death at $0.9 \%$, device failure at $0.9 \%$, and embolic events at $3 \%$.

Similar findings were observed with the electric HeartMate VE LVAD clinical trial.

TCI REGISTRY DATA. Although clinical trial data are reliable data, they are a limited snapshot of experience from the early phases of the field, when volumes were low, incorporating the first $10 \%$ of the total experience that has
Table 1. Patient Demographics, TCI Registry Data

\begin{tabular}{lccc}
\hline & $\begin{array}{c}\text { Pneumatic } \\
\text { (IP) } \\
\text { LVAD }\end{array}$ & $\begin{array}{c}\text { Electric } \\
\text { (VE) } \\
\text { LVAD }\end{array}$ & $\begin{array}{c}\text { Combined } \\
\text { IP + VE } \\
\text { LVAD }\end{array}$ \\
\hline Number of patients & 1,215 & 1,150 & 2,365 \\
Average age & 49 & 51 & 50 \\
Age range & $8-74$ & $11-78$ & $8-78$ \\
Males/females & $84 \%: 16 \%$ & $84 \%: 16 \%$ & $84 \%: 16 \%$ \\
Idiopathic CM & $52 \%$ & $49 \%$ & $51 \%$ \\
Ischemic CM & $41 \%$ & $44 \%$ & $43 \%$ \\
Acute MI & $4 \%$ & $4 \%$ & $4 \%$ \\
\hline
\end{tabular}

$\mathrm{CM}=$ cardiomyopathy; $\quad$ LVAD $=$ left ventricular assist device; MI = myocardial infarction

been accumulated to date. The best, and only, composite, up-to-date information available today comes from a Registry maintained by TCI. The data cover most of LVADs that have been implanted, with 2,365 included in the registry (data on file at TCI).

Characteristics of patients receiving HeartMate LVADs are listed in Table 1. Most of these patients fell into the bridge to transplant category, whereas some were bridged to recovery. Average age was 50 years old, ranging from 8 to 78 years. Eighty-four percent of recipients were male, with a large majority having chronic cardiomyopathies and a small number having acute myocardial infarctions.

Outcomes with HeartMate LVAD support are displayed in Table 2. Overall, $65 \%$ of patients experienced a successful outcome, living until the end of their LVAD experience when they were either transplanted or had their LVADs explanted after LV recovery. Once patients survived the first month after implantation, they had an $85 \%$ likelihood of a successful outcome, indicating that the majority of mortality occurs in the early postoperative period. Average duration of implant was 110 days, or 3.7 months, with the longest experience being 2.4 years.

Table 2. Patient Outcomes, TCI Registry Data

\begin{tabular}{lccc}
\hline & $\begin{array}{c}\text { Pneumatic } \\
\text { (IP) }\end{array}$ & $\begin{array}{c}\text { Electric } \\
\text { (VE) } \\
\text { LVAD }\end{array}$ & $\begin{array}{c}\text { Combined } \\
\text { IP + VE } \\
\text { LVAD }\end{array}$ \\
\hline $\begin{array}{c}\text { Number of patients } \\
\text { implanted }\end{array}$ & 1,215 & 1,150 & 2,365 \\
$\begin{array}{c}\text { Successful outcome } \\
\text { rate }\end{array}$ & $71 \%$ & $59 \%$ & $65 \%$ \\
$\begin{array}{c}\text { Success outcome } \\
\text { (after 1st 30 days) }\end{array}$ & $89 \%$ & $80 \%$ & $85 \%$ \\
$\begin{array}{c}\text { Average implant } \\
\text { duration }\end{array}$ & 91 days & 131 days & 110 days \\
$\begin{array}{c}\text { Maximum length of } \\
\text { support }\end{array}$ & 726 days & 884 days & 884 days \\
$\begin{array}{c}\text { Cumulative patient } \\
\text { experience }\end{array}$ & 291 years & 404 years & 695 years \\
\hline
\end{tabular}

a Successful outcome rate is the percentage of patients completing bridge to transplantation or to explant. Outcome after 30 days is the success rate for those patients who survived the immediate postoperative period.

LVAD $=$ left ventricular assist device. 
REPORTED RESUlTs. Published results from single centers, or small groups of centers, have been similar to the early clinical trial data and the TCI Registry. It has been well documented, repeatedly, that patients can be bridged to transplantation successfully with the HeartMate.

Most of the available information has focused on outcomes in patients with chronic cardiomyopathies, idiopathic and ischemic. Although experience has been more limited, patients with acute hemodynamic compromise, such as acute myocardial infarction and postcardiotomy cardiogenic shock, have also been shown to benefit from the HeartMate as a bridge to transplantation [14].

Not only has it been established that patients on HeartMates do better while awaiting transplantation, but it is now recognized that patients bridged with HeartMates do at least as well after transplantation as those that were not bridged. Patients supported with the HeartMate are in better shape at the time of transplantation and have improved survivorship after transplantation [15].

The overall mortality rates and the rates of complications during support with the HeartMate LVAD's occur with wide variation: mortality (on LVAD), $10 \%$ to $45+\%$; bleeding, $10 \%$ to $30 \%$; right heart failure, $5 \%$ to $20 \%$; embolism (thrombus or air), $2 \%$ to $5 \%$; infection, $5 \%$ to $40+\%$; renal, hepatic dysfunction, $30 \%$ to $60 \%$; immune sensitization, $20+\%$; device failure (requiring replacement), $1 \%$ to $5 \%$.

Complication rates vary considerably despite similar patient populations and identical technologies. The lower rates represent what may be possible. It is expected that complications will decrease with advances in clinical management. As the therapy is further established, less moribund patients will be implanted. What is clear, even now, is that once patients recover beyond their mortally ill states because of end-stage heart failure as well as the challenges of LVAD implantation, they can be managed with a low incidence of adverse events.

Once beyond the postoperative, inpatient period, when the majority of complications occur, long term outpatient management, which has become routine with the electric HeartMate VE, is safe with low levels of complications [16, 17].

Most of the outcomes with the HeartMate LVADs are generally considered to be comparable with the experience accumulated with other, similar technology. Two areas in which it has been variably suggested that there might be blood pump technology-related differences in clinical outcome include thromboembolism and device reliability.

Any discussion of differences between technologies must bear in mind that no strongly conclusive information is available by which different technologies may be compared with confidence. No fully controlled prospective, randomized, large-volume studies comparing devices have yet been undertaken. A limited amount of comparative information comes from individual center reports of small-volume experiences with multiple devices in the same center [18]. Even data of this type are limited because the information often reflects changing practice and experience over the course of time, using one device predominantly during one period of time followed by another device in a later period of time. Sorting out how much of the reported difference is from device factors and not clinical management factors is difficult, especially when reported complication rates exceed those observed in other centers.

Although statistically significant differences between devices have not been established, the following general observations can be made about thromboembolism. The TCI HeartMate experience has established a new "gold standard" for control of thromboembolism. The rate of thromboembolism in reports of TCI LVAD use has been consistently low, approximately $2 \%$ to $4 \%$ [19]. This is at least as good as would be expected in similar heart failure patients without an LVAD. Remarkably, this experience occurs with minimal anticoagulation, low-level antiplatelet therapy only, a benefit no other device at present can offer.

On the other hand, the perception exists that the HeartMate VE LVAD may experience limitations with long-term reliability. One way to examine this issue is to evaluate rates of device exchange for device failure.

Rates of device exchange for malfunction of the VE LVAD have been reported as $2 \%$ [20] and 6\% [21], whereas many centers, especially those with lower volumes, have not yet experienced these complications. In a few cases, device failures have been associated with mortality [21, 22]. Most often, however, device failures are not associated with catastrophic outcomes. Generally, device failures can be compensated for or resolved with device replacement with a satisfactory degree of success [16].

The pneumatic HeartMate IP has experienced less device failure that the electric VE. One center has chosen to use the pneumatic HeartMate for long-term use, despite a cumbersome external driver, over the selfcontained electric VE HeartMate, primarily because of expected better device reliability. This approach does not reflect general preference, however.

Device failure-related adverse events have been approached as an opportunity for improvement. Data from TCI indicate that overall average annual device exchange rate from 1993 to 1999 was $2.4 \%$. Reliability is improving as device modifications have been made, approaching reasonable levels. The device failure rate has decreased from a high of a $4.6 \%$ device exchange rate for malfunction in 1995 to $1.0 \%$ and $1.1 \%$ in 1998 and 1999. Additional recent device improvements may decrease this further (data on file at TCI).

Although premature device failures are decreasing, as the number of patients on extended support beyond 2 years increases, it is expected that more replacements for "end-of-life" will be required. Presently, it is not possible to predict an expected lifetime for the HeartMate VE first-generation electric LVAD beyond 2 to 3 years. In vitro reliability testing, still ongoing, has established that 12 of 15 devices continue functioning at 3 years, with three devices failing at 1.1, 2.3, and 2.7 years.

Although there is room to improve device reliability, in the final, bottom-line analysis, the TCI HeartMate has 
delivered a level of reliability sufficient to prove its effectiveness and safety for bridging to transplantation. It is expected by many that it will be good enough to be effective as an alternative to transplantation, at least until next-generation devices with improved reliability have been proved and become widely accepted.

In addition to reports of outcomes analyzing hemodynamic or end-organ effectiveness and reports of safety, other studies have focused on scientific and systemic issues. A fascinating view of the immunobiology of the HeartMate and its potential relevance to infection has been reported [23]. Patients with HeartMate LVADs have somewhat lower exercise capacity while LVADs are implanted than they do after they have been transplanted and the LVAD removed. This information may be of relevance to the design of next-generation devices [24].

\section{Comment}

HeartMate LVADs, as first-generation technology, have played a leading role, giving clinicians the opportunity to demonstrate successful management of heart failure with advanced mechanical circulatory support.

Survival, at least with bridging to transplantation, is clearly improved with the HeartMate LVAD's. Beneficial outcomes with "permanent" use and bridging to recovery appear possible.

Complication rates have been reasonable given the high-risk patient population and the early learning curve of the field. Improvements are expected as patient selection and management evolves.

Strokes and thromboembolic events occur with remarkable infrequency. The success of aspirin-only anticoagulation is encouraging. The level of thromboresistance afforded by the HeartMate is an important "gold standard" for all advanced mechanical circulatory support devices.

Quality of life while supported with a HeartMate has improved substantially since its introduction with patients 15 years ago. No longer must patients with HeartMate LVADs be tethered to large, external consoles or confined to hospitals. Patients are returning to their homes often in communities distant from the implanting center. As they return to productive lives, airline travel and driving are being introduced cautiously. However, several limitations remain and offer potential opportunities for advancement. The effectiveness of the HeartMate as a "permanent" alternative to transplantation or as a bridge to recovery has yet to be fully established. Enthusiasm for these indications remains high. The permanent, alternative to transplantation indication is being studied rigorously in the REMATCH trial. The study of left ventricular recovery is still immature and much has to be learned about predicting and optimizing sustainable recovery.

Small adults and children cannot be treated with the HeartMate because of its large size. Even for larger recipients, the presence of the pump can limit full mobility and can compress on intraabdominal structures.
Size and component complexity add to the challenges of device removal or exchange.

Device reliability will limit the use of this technology to 2- to 3-year durations, after which end-of-life replacement will be expected. Longer term support is required to generate more value for the purchasers of this technology.

The percutaneous lead with external venting allowed early use of the HeartMate in patients. However, large diameter and stiffness of the lead have made immobilization to prevent exit site trauma and breakdown difficult. Improvements with a smaller, more flexible, infection-resistant lead are required.

Infections, as with all devices, remain a significant challenge. Improvements in the percutaneous lead system as well as its clinical management (eg, immobilization, exit site care) and eventual elimination of a percutaneous lead will reduce this problem. Further advancements in clinical practice with prevention and management could play an even greater role to minimize the threat of infection.

Noise, as little as is produced with any first-generation device, is generally well tolerated, but improvement, especially for low-noise environments, would be helpful. Similarly, vibration and movement from motor torque is usually not a problem, but is bothersome to some patients, especially at high flow rates. Battery life is a limitation, requiring exchange every 3 to 7 hours, depending on wear. In addition to technologic factors, advancements in independent, outpatient management will add to quality of life.

Configuration and size considerations preclude use of the HeartMate for right heart support. The clinical courses of perhaps $15 \%$ to $20 \%$ of patients could be improved with implantable right heart mechanical assistance, a capability that will have to await next-generation technology.

Most of these issues are common to all first-generation, implantable technologies and represent challenges to both the developmental and clinical communities.

\section{References}

1. Poirier VL. The heartmate left ventricular assist system: worldwide clinical results. Eur J Cardiothorac Surg 1997; 11(Suppl):S39-44.

2. Poirier VL. Worldwide experience with the TCI HeartMate system: issues and future perspective. Thorac Cardiovasc Surg 1999;47(Suppl 2):316-20.

3. Graham TR, Dasse K, Coumbe A, et al. Neo-intimal development on textured biomaterial surfaces during clinical use of an implantable left ventricular assist device. Eur J Cardiothorac Surg 1990;4:182-90.

4. Frazier OH, Baldwin RT, Eskin SG, et al. Immunochemical identification of human endothelial cells on the lining of a ventricular assist device. Tex Heart Inst J 1993;20:78-82.

5. Rose EA, Levin HR, Oz MC, et al. Artificial circulatory support with textured interior surfaces. A counterintuitive approach to minimizing thromboembolism. Circulation 1994;90:II87-91.

6. Rafii S, Oz MC, Seldomridge JA, et al. Characterization of hematopoietic cells arising on the textured surface of left ventricular assist devices. Ann Thorac Surg 1995;60:1627-32. 
7. Spanier TB, Chen JM, Oz MC, et al. Time-dependent cellular population of textured-surface left ventricular assist devices contributes to the development of a biphasic systemic procoagulant response. J Thorac Cardiovasc Surg 1999;118: 404-13.

8. Rose EA, Moskowitz AJ, Packer M, et al. The REMATCH trial: rationale, design, and end points. Randomized Evaluation of Mechanical Assistance for the Treatment of Congestive Heart Failure. Ann Thorac Surg 1999;67:723-30.

9. Helman DN, Addonizio LJ, Morales DL, et al. Implantable left ventricular assist devices can successfully bridge adolescent patients to transplant. J Heart Lung Transplant 2000;19: 121-6.

10. Oz MC, Goldstein DJ, Pepino P, et al. Screening scale predicts patients successfully receiving long-term implantable left ventricular assist devices. Circulation 1995;92(Suppl 9):II169-73.

11. McCarthy PM, Wang N, Vargo R. Preperitoneal insertion of the HeartMate 1000 IP implantable left ventricular assist device. Ann Thorac Surg 1994;57:634-8.

12. Frazier OH, Duncan JM, Radovancevic B, et al. Successful bridge to heart transplantation with a new left ventricular assist device. J Heart Lung Transplant 1992;11:530-7.

13. Tector AJ, Kress DC, Downey FX, et al. Transition from cardiopulmonary bypass to the HeartMate left ventricular assist device. Ann Thorac Surg 1998;65:643-6.

14. Helman DN, Rose EA. History of mechanical circulatory support. Prog Cardiovasc Dis 2000;43:1-4.

15. Bank AJ, Mir SH, Nguyen DQ, et al. Effects of left ventricular assist devices on outcomes in patients undergoing heart transplantation. Ann Thorac Surg 2000;69:1369-75.

16. Morales DL, Catanese KA, Helman DN, et al. Six-year experience of caring for forty-four patients with a left ven- tricular assist device at home: safe, economical, necessary. J Thorac Cardiovasc Surg 2000;119:251-9.

17. Schmid C, Hammel D, Deng MC, et al. Ambulatory care of patients with left ventricular assist devices. Circulation 1999; 100(Suppl 19):II224-8.

18. El-Banayosy A, Arusoglu L, Kizner L, et al. Novacor left ventricular assist system versus HeartMate vented electric left ventricular assist system as a long-term mechanical circulatory support device in bridging patients: a prospective study. J Thorac Cardiovasc Surg 2000;119:581-7.

19. Slater JP, Rose EA, Levin HR, et al. Low thromboembolic risk without anticoagulation using advanced-design left ventricular assist device. Ann Thorac Surg 1996;62:1321-8.

20. Sun BC, Catanese KA, Spanier TB, et al. 100 long-term implantable left ventricular assist devices: the Columbia Presbyterian interim experience. Ann Thorac Surg 1999;68: 688-94.

21. McCarthy PM, Smedira NO, Vargo RL, et al. One hundred patients with the HeartMate left ventricular assist device: evolving concepts and technology. J Thorac Cardiovasc Surg 1998;115:904-12.

22. Bonkohara Y, Minami K, Arusoglu L, et al. A fatal mechanical disorder of the TCI HeartMate left ventricular assist system [Letter]. J Thorac Cardiovasc Surg 1999;118:769-70.

23. Itescu S, Ankersmit JH, Kocher AA, et al. Immunobiology of left ventricular assist devices. Prog Cardiovasc Dis 2000;43: 67-80.

24. Jaski BE, Lingle RJ, Kim J, et al. Comparison of functional capacity in patients with end-stage heart failure following implantation of a left ventricular assist device versus heart transplantation: results of the experience with left ventricular assist device with exercise trial. J Heart Lung Transplant 1999;18:1031-40. 